\title{
Importance of appropriate initial antibiotic therapy and de-escalation in the treatment of nosocomial pneumonia
}

\author{
J. Rello
}

ABSTRACT: Inappropriate initial antibiotic therapy in nosocomial pneumonia is associated with higher mortality, longer hospital stays and increased healthcare costs. The key pathogens associated with these adverse outcomes include Pseudomonas aeruginosa, methicillin-resistant Staphylococcus aureus and Acinetobacter baumannii.

Due to the increasing rates of resistance, a new paradigm is needed for treating nosocomial infections in the intensive care unit (ICU). Optimal initial therapy consists of a broad-spectrum antibiotic started in a timely manner and administered at the correct dose and via the correct route.

Because pathogen aetiology and resistance patterns vary from one ICU to another, recommendations for initial therapy should be tailored to each institution. Selection of the broad-spectrum antibiotic should be based on the patient's risk factors (including comorbidities, duration of ventilation and recent antibiotic exposure), suspected pathogen and up-to-date local resistance patterns.

After 48-72 $\mathrm{h}$, the patient should be reassessed and antibiotic therapy de-escalated based on the microbiological results and the clinical response.

KEYWORDS: Appropriate antibiotic therapy, broad-spectrum antibiotics, carbapenems, nosocomial pneumonia, ventilator-associated pneumonia

$\mathbf{T}$ he increasing emergence of antimicrobial resistance in the intensive care unit (ICU) threatens patient outcomes and substantially raises overall healthcare costs [1]. Key nosocomial pathogens that were once susceptible to commonly used antibiotics are now showing alarming rates of resistance. The National Nosocomial Infections Surveillance System reported that, in the USA in 2003, methicillin resistance was identified in $59.5 \%$ of ICU patients with nosocomial infections caused by Staphylococcus aureus, i.e. an $11 \%$ increase over the preceding 5-yr period [2]. Furthermore, the rates of resistance to third-generation cephalosporins were $32 \%$ in Pseudomonas aeruginosa, 31\% in Enterobacter spp. and $20 \%$ in Klebsiella pneumoniae. Notably, the 2003 rates in P. aeruginosa and K. pneumoniae were 20 and $47 \%$ higher, respectively, than the corresponding resistance rates for the preceding 5-yr period.

Resistance rates measured in surveillance programmes are not only of academic interest, they have considerable importance in the clinical ICU setting. The impact of ventilator-associated pneumonia (VAP) on morbidity, mortality and healthcare costs is well recognised but, as illustrated in clinical studies, adverse outcomes are more likely when VAP is associated with resistant pathogens. Even among patients who received appropriate initial antibiotic therapy, the 28-day mortality rate was significantly higher when VAP was caused by piperacillin-resistant strains of $P$. aeruginosa than by piperacillin-susceptible strains (37 versus $19 \%, \mathrm{p}=0.04$ ). Additionally, the median length of hospital stay was significantly longer when VAP was caused by methicillin-resistant $S$. aureus (MRSA) than by methicillin-susceptible strains (33 versus 22 days, $p=0.047) \quad[3,4]$. Extended-spectrum $\beta$-lactamase (ESBL)-producing K. pneumoniae and Enterobacter spp. have also been associated with prolonged hospital stays, higher hospital costs and greater mortality $[5,6]$. For example, in a retrospective cohort of 147 patients with bloodstream infections caused by $K$. pneumoniae, ESBL-producing strains increased the mortality risk by $\sim 2$.6-fold as compared with nonESBL-producing strains $(p \leqslant 0.03)$ [7]. Furthermore, the presence of an ESBL-producing strain prolonged the hospital stay by an average of 6 days $(p=0.03)$ [7]. Thus, addressing antimicrobial resistance remains a top priority, both in clinical research and in patient management.
CORRESPONDENCE

J. Rello

Critical Care Dept

Joan XXIII University Hospital and CIBER Enfermedades Respiratorias Carrer Mallafre Guasch 4 43007 Tarragona Spain

Fax: 34977295878

E-mail: jrello.hj23.ics@gencat.net

STATEMENT OF INTEREST

J. Rello has received fees for consulting from Merck, JanssenCilag, Astra Zeneca and Pfizer. He is a member of the speaker bureau at the aforementioned companies. J. Rello has served as an expert witness on the subject of this study. 


\section{RISK ASSOCIATED WITH INAPPROPRIATE INITIAL ANTIBIOTIC THERAPY}

The choice of initial (empiric) antibiotic therapy can have a significant impact on patient outcome when resistant pathogens may be involved. The relationship between initial therapy and outcome is illustrated by a prospective cohort study of 2,000 consecutive patients admitted over 8 months to a 19-bed medical ICU and an 18-bed surgical ICU [8]. The adequacy of antibiotic therapy was determined after culture and susceptibility data became available. Inappropriate antibiotic therapy was defined as the use of an antimicrobial to which the documented pathogen was resistant, or as the failure to provide coverage against an identified pathogen. In the study cohort, 655 patients had a clinically recognised infection during their ICU stay, of which 169 (25.8\%) had received inappropriate initial antibiotic therapy. The primary reason for administration of inappropriate antibiotic therapy was the presence of antibiotic-resistant Gram-negative or Gram-positive bacteria that were not susceptible to the prescribed antibiotic regimen. Notably, in-hospital mortality was substantially higher among patients who received inappropriate initial therapy compared with those treated appropriately (52 versus $12 \%, \mathrm{p}<0.001)$. On multivariate analysis, inappropriate antibiotic therapy was the most important independent risk factor for in-hospital mortality, with an odds ratio of 4.26 (95\% confidence interval (CI) 3.35-5.44, p<0.001). In the study cohort, inappropriate antibiotic therapy was significantly more likely in patients with nosocomial infections that developed after the treatment of a community-acquired infection (45\%) than in those with nosocomial $(34 \%)$ or community-acquired infections $(17 \%, \mathrm{p}<0.001)$. Similarly, the likelihood of acquiring an antibiotic-resistant Gram-negative or Gram-positive pathogen was at least twice as high in patients with nosocomial infections than in those with community-acquired infections $(\mathrm{p}<0.001)$.

Figure 1 shows the results of several other studies that have demonstrated that inappropriate antibiotic therapy for ICU patients is associated with increased mortality. In a prospective multicentre study conducted in the medical and surgical ICUs of 30 Spanish hospitals over $1 \mathrm{yr}$, mortality attributable to pneumonia was significantly higher in patients with inappropriate initial antibiotic therapy than in those who received appropriate therapy ( 25 versus $16 \%, p=0.034$ ) [9]. Following the receipt of culture and susceptibility data, modification of the empirically used antibiotic was needed in $44 \%$ of pneumonia episodes. Similarly, in a cohort of 113 consecutive adults who developed VAP, infection-related mortality was significantly higher among patients with inappropriate initial antibiotic therapy than in those receiving appropriate therapy (37 versus $16 \%, \mathrm{p}<0.05$ ) [10]. All-cause mortality tended to be higher in patients receiving inappropriate initial therapy (63 versus $41 \%$, $\mathrm{p}=0.06$ ) [10]. In another cohort of $50 \mathrm{VAP}$ patients who received antibiotic therapy before bronchoscopy with bronchoalveolar lavage was performed, the mortality rate was $91 \%$ in patients receiving inadequate therapy, compared with $38 \%$ in patients receiving adequate antibiotics $(p<0.001)$ [11]. Higher mortality rates were also associated with inadequate initial therapy in patients admitted to the ICU with bloodstream infections, particularly in the presence of septic shock or in cases with greater illness severity [12-14].

\section{INCREASING ANTIBIOTIC RESISTANCE REQUIRES A NEW TREATMENT APPROACH}

The traditional approach to antibiotic therapy involved the initial use of a narrow-spectrum antibiotic, with the most potent drugs reserved for severely immunocompromised patients, patients who do not respond to treatment or are infected with a resistant pathogen. This approach is based on the desire to avoid antibiotic exposure when infection is not confirmed, limiting the development of resistance and allowing the control of costs. The traditional approach, however, may no longer be appropriate in the current era of increasing antibiotic resistance. In the aforementioned studies, it is important to recognise that the excess mortality associated with inadequate initial therapy occurred even though the antibiotic could be switched once culture and susceptibility data became available. The delay may have been only $2-3$ days but, by that time, it was already too late in many cases.

These data underscore the need for a new approach to treating nosocomial infections: one that focuses on prescribing optimal antibiotic therapy and not just appropriate or adequate therapy, as shown in figure 2. Optimal therapy depends on the pharmacokinetic-pharmacodynamic relationships between antibiotics and the key nosocomial pathogens [15, 16]. In contrast, appropriate antibiotic therapy only considers whether the selected antibiotic provides pathogen coverage. In the optimisation of the initial therapy, antibiotics should be selected that provide coverage of the key pathogens, including resistant strains, and treatment should be initiated in a timely manner using the correct dose and the correct route of administration to ensure that sufficient antibiotic concentrations are achieved at the site of infection. For example, the antibiotic concentration achieved in the epithelial lining fluid and in alveolar macrophages is relevant to the optimal therapy of pneumonia caused by extracellular and intracellular pathogens, respectively.

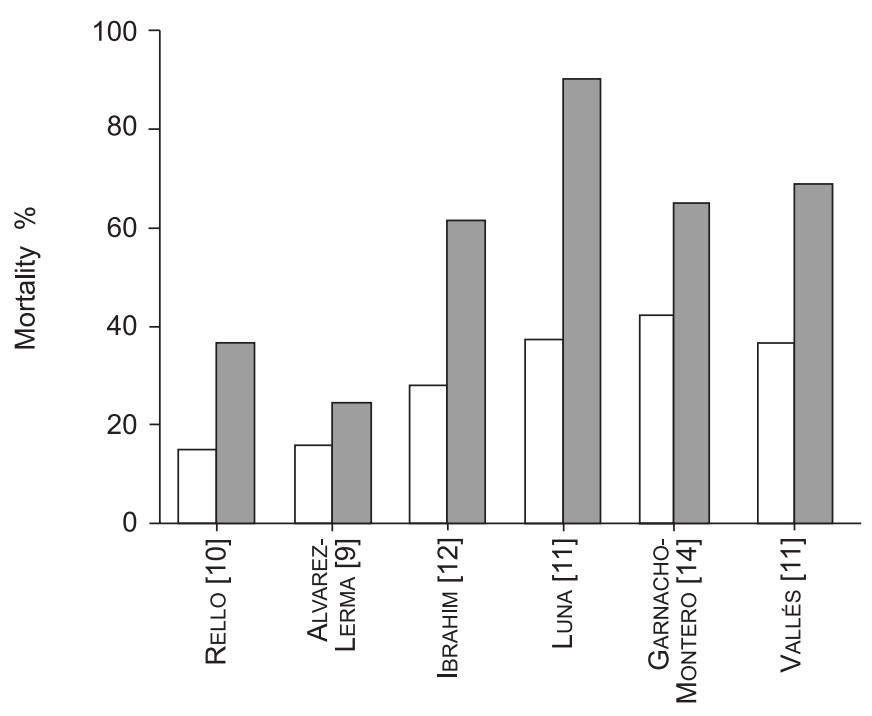

FIGURE 1. Association between inappropriate initial antibiotic therapy and higher mortality rates in studies of patients with nosocomial pneumonia or bloodstream infections. $\square$ : appropriate therapy; $\square$ : inappropriate therapy. 


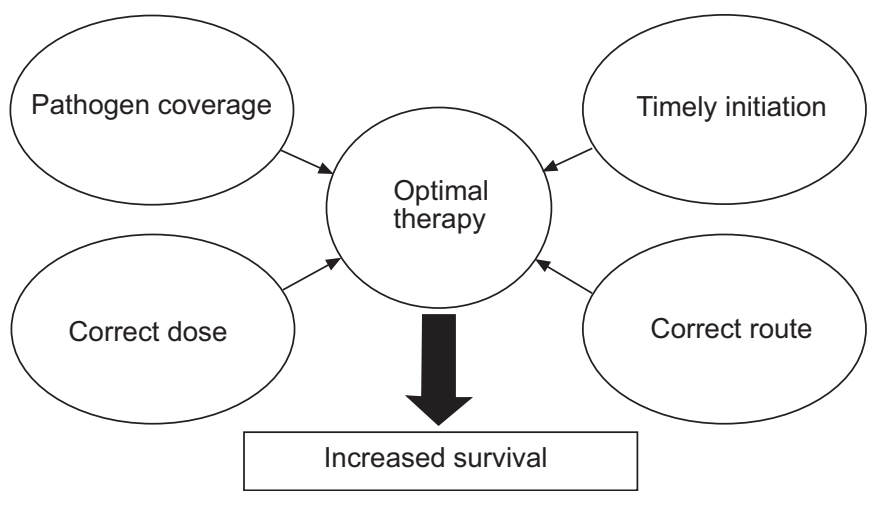

FIGURE 2. Factors involved in optimal initial antibiotic therapy.

\section{KEY PATHOGENS IN VAP}

Only a limited number of pathogens are significantly involved in VAP. By focusing on patients who fail initial antibiotic therapy and ultimately die from their infection, P. aeruginosa, MRSA and Acinetobacter baumannii emerge as the three most important. Both $P$. aeruginosa and A. baumannii are nonfermentative Gram-negative pathogens that cause mortality in 30-75\% of nosocomial pneumonia patients, with the highest rates reported in those who are ventilator-dependent [17]. For $P$. aeruginosa, the most important risk factors are extended ICU stay, prolonged mechanical ventilation and recent exposure to antimicrobial therapy. The presence of adult respiratory distress syndrome (ARDS) and severe chronic obstructive pulmonary disease (COPD) also independently increase the risk of $P$. aeruginosa infection. Based on a prospective study of 707 mechanically ventilated patients in three medical ICUs in teaching hospitals in Uruguay and Spain [18], the risk of VAP due to $A$. baumannii appears independently associated with neurosurgery, ARDS, head trauma and large-volume pulmonary aspiration. The presence of comorbid illness and recent exposure to antibiotics, however, did not appear to predict $A$. baumannii infection; therefore, risk factors for these nonfermentative Gram-negative pathogens differ.

MRSA is the most important Gram-positive pathogen in VAP. When all episodes of VAP caused by $S$. aureus in two Spanish hospitals over a 30-month period were analysed prospectively, the risk of MRSA infection was associated with recent corticosteroid treatment, ventilation for $>6$ days, age $>25$ yrs and the presence of COPD [19]. In contrast, patients with methicillin-susceptible $S$. aureus (MSSA) were more likely to have head trauma. Notably, all patients with MRSA, but only $21 \%$ of those with MSSA, had recently been treated with antibiotics $(\mathrm{p}<0.001)$. Thus, taken together, the overriding factors associated with infection by the key VAP pathogens, particularly $P$. aeruginosa and MRSA, are prolonged mechanical ventilation and recent antibiotic therapy. By avoiding unnecessary antibiotic exposure and by promptly initiating optimal antibiotic therapy, it may be possible to limit infection by these pathogens and improve patient outcomes.

\section{INITIAL ANTIBIOTIC THERAPY}

According to the American Thoracic Society and the Infectious Diseases Society of America, patients at risk of infection by antibiotic-resistant pathogens should initially be treated with antibiotics that provide a broad spectrum of coverage in order to minimise the potential for inappropriate therapy [20]. Initial antibiotic therapy recommended for nosocomial pneumonia or VAP caused by $P$. aeruginosa or $A$. baumannii include an antipseudomonal $\beta$-lactam, such as piperacillin-tazobactam, a carbapenem or a third- or fourth-generation cephalosporin, together with either an aminoglycoside or an antipseudomonal fluoroquinolone. The initial therapy recommended for MRSA is vancomycin or linezolid. In a post hoc analysis of a subset of 160 patients with documented MRSA pneumonia who participated in two randomised, double-blind studies, linezolid was shown to provide significantly higher clinical cure rates ( 59.0 versus $35.5 \%, \mathrm{p}<0.01)$ and survival rates (80.0 versus $63.5 \%, \mathrm{p}=0.03)[21]$.

Cephalosporins are becoming increasingly inappropriate for the initial therapy of nosocomial pneumonia, since the most common aetiological agents, including $P$. aeruginosa, S. aureus, Enterobacter spp., Klebsiella spp., A. baumannii, Haemophilus influenzae and Streptococcus pneumoniae, are often resistant [22, 23]. Moreover, even if a pathogen appears susceptible, thirdgeneration cephalosporins are prone to select for resistant mutants. In nosocomial pneumonia, resistance is often caused by the production of ESBLs or Ampicillin resistance gene C $(\mathrm{AmpC}) \beta$-lactamases. ESBLs are a heterogeneous group of plasmid-encoded $\beta$-lactamases, which are mainly produced by Klebsiella spp., Escherichia coli and Proteus spp., that are currently being detected with increasing frequency in $P$. aeruginosa and Enterobacter spp. [24]. The AmpC $\beta$-lactamases, also known as the group 1 or Ambler class $C \beta$-lactamases, are encoded by genes found on the bacterial chromosome in $P$. aeruginosa and a number of other Gram-negative organisms [25]. However, AmpC has also been identified on plasmids and shown to be transferable to other Gram-negative organisms, most commonly K. pneumoniae and E. coli [26].

The ESBLs can elevate the minimum inhibitory concentration (MIC) of third-generation cephalosporins and aztreonam, and sometimes cefepime as well, as illustrated in table 1. In comparison, AmpC expression conveys resistance to the third-generation cephalosporins as well as many $\beta$-lactamase inhibitors, although strains producing these enzymes remain susceptible to cefepime [25]. Because ESBLs are encoded by plasmids, and AmpC can also be carried on plasmids, strains that produce these enzymes are often cross-resistant to other antibiotics, including aminoglycosides and fluoroquinolones. For example, fluoroquinolone resistance has been identified in $18-56 \%$ of ESBL-producing strains of Klebsiella spp. [27-29]. In surveys conducted in ICUs in western and southern Europe, the prevalence of ESBL-producing strains of Klebsiella spp. (23-25\%) did not change appreciably in the period 1994-1998, but the proportion of ESBL-producing strains that were resistant to piperacillin/tazobactam rose significantly from 31 to $63 \%(p<0.001)$ [28]. Most of the strains showed high-level resistance to piperacillin/tazobactam, with MICs $>128 \mathrm{mg} \cdot \mathrm{L}^{-1}$. Notably, a substantial proportion of piperacillin/tazobactamresistant strains of $P$. aeruginosa isolated from VAP patients showed cross-resistance to other antibiotic classes, including third-generation cephalosporins (62\%), fluoroquinolones $(71 \%)$, carbapenems (59\%) and aminoglycosides (50\%) [30]. 


\begin{tabular}{|c|c|c|c|}
\hline \multirow{2}{*}{$\begin{array}{l}\text { TABLE } 1 \\
\text { Antibiotic }\end{array}$} & \multicolumn{3}{|c|}{$\begin{array}{l}\text { Adequate therapy for } \beta \text {-lactamase-producing } \\
\text { pathogens }\end{array}$} \\
\hline & & ESBL & AmpC \\
\hline \multicolumn{2}{|c|}{ Third-generation cephalosporins } & - & - \\
\hline \multicolumn{2}{|c|}{ Cefepime } & - & ++ \\
\hline \multicolumn{2}{|c|}{ Fluoroquinolones } & $+/-$ & + \\
\hline \multicolumn{2}{|c|}{ Piperacillin/tazobactam } & $+/-$ & $+/-$ \\
\hline \multicolumn{2}{|c|}{ Tigecycline } & ++ & ++ \\
\hline \multicolumn{2}{|c|}{ Carbapenem } & +r+ & +r+ \\
\hline
\end{tabular}

ESBL: extended-spectrum $\beta$-lactamases; AmpC: Ampicillin resistance gene $\mathrm{C}$. -: not effective; +: effective; ++: more effective; ++t: most effective; +/-: effective against some, but not all, $\beta$-lactamases.

Carbapenems provide a broad spectrum of activity against the Gram-positive and Gram-negative pathogens encountered in serious nosocomial infections [31, 32]. These agents remain active against $\beta$-lactamase-positive pathogens, including those producing ESBLs and AmpC $\beta$-lactamases. Despite their increasing usage, surveillance studies including the Meropenem Yearly Susceptibility Test Information Collection and the SENTRY Antimicrobial Surveillance Program continue to highlight the low level of resistance to carbapenems, particularly in the ICU setting [33]. The carbapenems are particularly appropriate in initial therapy for late-onset nosocomial pneumonia and when multidrug-resistant pathogens, including ESBLs or AmpC $\beta$-lactamase-producing strains, are suspected [32]. In addition, carbapenems are appropriate for patients with nosocomial pneumonia who are at increased risk of infection with multidrug-resistant pathogens, including recently hospitalised patients, nursing home residents and those recently treated with antibiotics. Due to their broad spectrum of activity, carbapenems are also appropriate in second-line therapy after the failure of previous therapy with cephalosporins, aminoglycosides or fluoroquinolones. However, as noted previously, the selection of optimal initial antibiotic therapy is critical to patient outcome and, therefore, reserving carbapenems for second-line treatment may be too late to prevent death in many cases.

\section{TAILORING THERAPY IN EACH INSTITUTION}

When considering initial antibiotic therapy for VAP, national and regional guidelines should be modified on the basis of upto-date information about pathogen aetiology and antibiotic resistance patterns in each institution. This critical point is illustrated in a retrospective study comparing VAP aetiology in four ICUs in France, Spain and Uruguay [34]. Patients were stratified into four groups on the basis of the duration of ventilation ( $<7$ days versus $\geqslant 7$ days), as well as antibiotic use in the previous 15-day period. In all four groups, the causes of VAP varied markedly across the four ICU sites. For example, the frequency of $P$. aeruginosa ranged: $12-66 \%$ in VAP patients ventilated for $<7$ days and exposed recently to antibiotics; 6$43 \%$ in patients ventilated for $\geqslant 7$ days and not treated with antibiotics; and $22-86 \%$ in patients ventilated for $\geqslant 7$ days and exposed to antibiotics. Similarly, one site had a much lower incidence of MRSA than the other sites. The variation in key pathogens across sites is also illustrated in figure 3 , which shows the distribution of $P$. aeruginosa, MRSA, Acinetobacter spp. and Stenotrophomonas maltophilia among patients with lateonset VAP who had been recently exposed to antibiotics [35]. The observed differences in pathogen aetiology across institutions reflect differences in patient demographics, diagnostic methods, antibiotic use and local patterns of resistance.

The differences in pathogen aetiology and resistance patterns across ICUs prompted the development of a more patientspecific antibiotic management programme at the present author's institution; The Tarragona Strategy for VAP [36]. The treatment algorithm is shown in figure 4 . In cases of suspected VAP, a broad-spectrum antibiotic should be started immediately. The selection of initial antibiotic therapy should be based on the patient's risk factors, including comorbidities, duration of ventilation and recent antibiotic exposure, as well as on local pathogen resistance patterns. Courses of therapy should be given at high doses according to the pharmacodynamic and tissue penetration properties. A combination of two antipseudomonal agents should be used when $P$. aeruginosa is suspected; a carbapenem should be given when A. baumannii is suspected; and linezolid should be provided when MRSA is suspected. The patient should be reassessed at $48-72 \mathrm{~h}$ and antibiotic therapy should be de-escalated based on the microbiological results and clinical response. De-escalation includes changing from the broad-spectrum antibiotic to an agent with a narrow focus, based on the culture data; changing the focus from multiple antibiotics to a single drug when $P$. aeruginosa is not detected by culture; and shortening the course of therapy to $<5$ days in cases with negative culture results and $\geqslant 48 \mathrm{~h}$ without fever [35]. Accordingly, the same microbiology report may lead to different actions depending on the clinical response. When ARDS is not present, early clinical resolution of fever and hypoxaemia may be expected in $\sim 75 \%$ of patients. When ARDS is present, however, clinical resolution is seen in a median of 6 days.

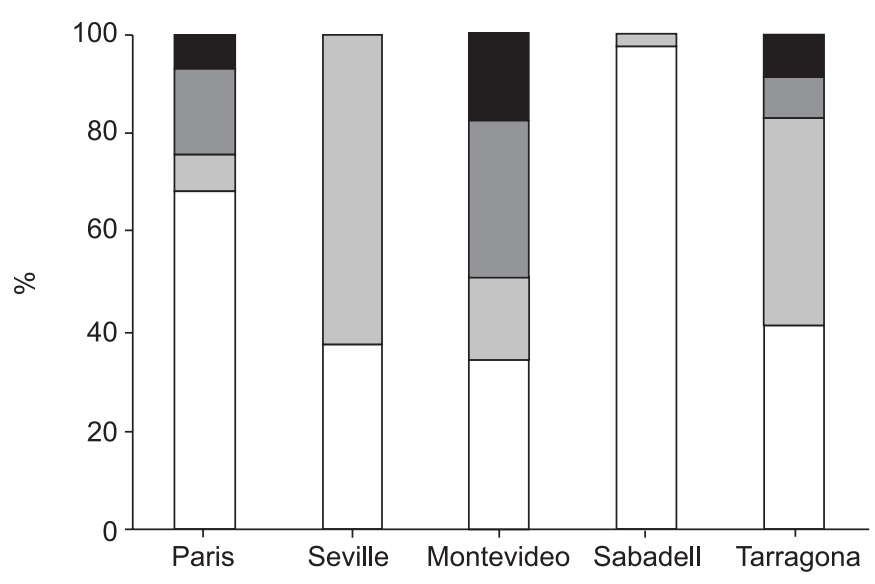

FIGURE 3. Variation in causative pathogens in ventilator-associated pneumonia in five different intensive care units. $\square$ : Pseudomonas aeruginosa; 1 : : methicillinresistant Staphylococcus aureus; $\mathbf{\square}:$ Acinetobacter; 口: Stenotrophomonas maltophilia. Reproduced from [35] with permission from the publisher. 


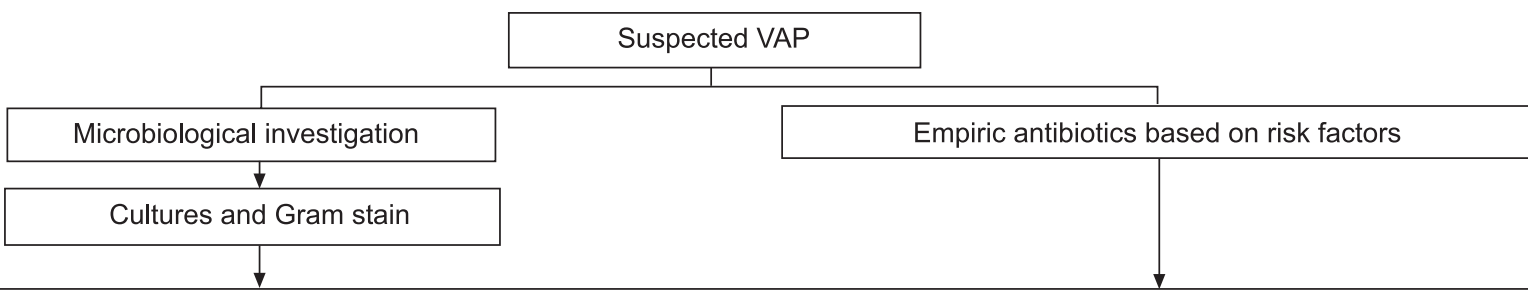

Gram-positive stain: if MRSA, start anti-MRSA coverage

Gram-negative stain: if Acinetobacter baumannii, start carbapenem; if Pseudomonas species, start two antipseudomonal agents If none of the above, start antibiotics and consider local epidemiology

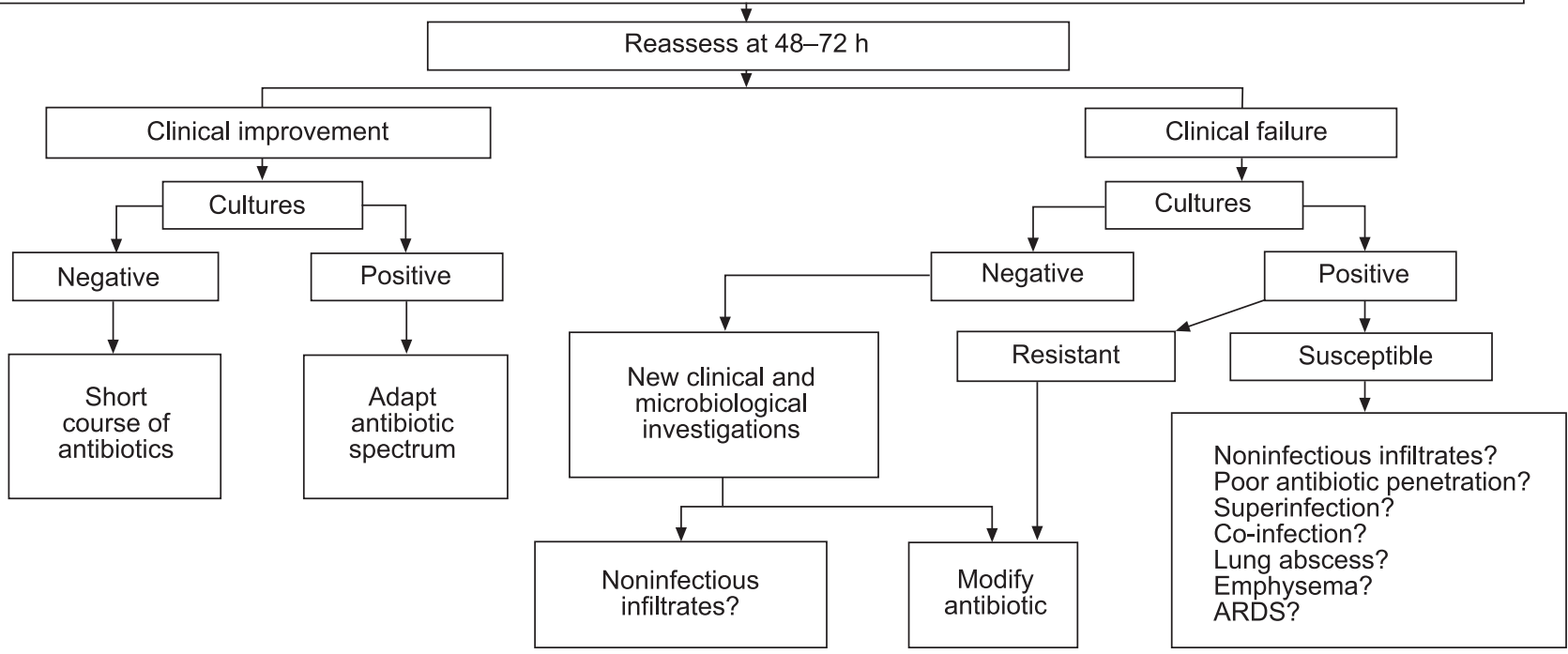

FIGURE 4. Treatment algorithm for ventilator-associated pneumonia (VAP). MRSA: methicillin-resistant Staphylococcus aureus; ARDS: adult respiratory distress syndrome. Reproduced from [23] with permission from the publisher.

\section{NEWLY APPROVED AND INVESTIGATIONAL ANTIMICROBIAL AGENTS}

Many newly approved and investigational agents for nosocomial pneumonia have been developed recently. Most focus on Gram-positive pathogens and include the oxazolidinones (linezolid, ranbezolid and DA-7867), streptogramins (quinupristindalfopristin) and glycopeptides (telavancin, dalbavancin, oritavancin and stenavancin). Only a few agents, specifically tigecycline and the investigational agents ceftobiprole and doripenem, focus on Gram-negative pathogens.

Tigecycline is a member of the glycylcycline class, which was developed to avoid tetracycline resistance mediated by ribosomal protection and drug efflux [37]. Like the tetracyclines, this agent inhibits bacterial protein synthesis by binding to the $30 \mathrm{~S}$ ribosomal subunit. Tigecycline is active against MRSA, ESBLproducing Gram-negative organisms, Acinetobacter spp. and $S$. maltophilia. Unfortunately, it is inactive against organisms with chromosomal efflux pumps, such as $P$. aeruginosa and Proteus spp. Tigecycline is currently being compared with imipenem/ cilastin in a phase III study in nosocomial pneumonia.

Ceftobiprole, an investigational cephalosporin, has activity against MRSA, as well as penicillin-resistant S. pneumoniae, many ESBL-producing Gram-negative bacteria and P. aeruginosa
[38]. It is currently in phase III trials for nosocomial pneumonia, skin and skin-structure infections and community-acquired pneumonia. In the future, if there are several $\beta$-lactams with excellent activity against MRSA, it is likely that they will be the optimal therapy for MRSA.

Doripenem, an investigational carbapenem, has a broad spectrum of activity against Gram-positive and Gram-negative organisms. This agent is active against ESBL-producing strains, as well as the nonfermentative Gram-negative pathogens $P$. aeruginosa and $A$. baumannii. There are few other options for Gram-negative infections in development. For example, tigecycline and the new glycopeptides all have poor activity against $P$. aeruginosa. Doripenem differs from other carbapenems in several ways. First, it exhibits enhanced activity against $P$. aeruginosa. Against a panel of 3,330 P. aeruginosa isolates collected in the period 2003-2005, doripenem inhibited more strains than either meropenem or imipenem at a breakpoint of $\leqslant 4 \mathrm{mg} \cdot \mathrm{L}^{-1}$, regardless of whether the strains were collected in Europe, Latin America or North America [39]. Secondly, in vitro studies suggest that doripenem has a lower propensity than the other carbapenems for developing resistance in $P$. aeruginosa [40, 41]. Thirdly, unlike other carbapenems, doripenem did not show a potential for seizures in experimental models [42]. Finally, doripenem is more stable 
after reconstitution, allowing administration via extended i.v. infusions which, in turn, have the potential to maximise the time $>$ MIC, the key pharmacokinetic/pharmacodynamic parameter of carbapenem activity. Doripenem has completed phase III trials for complicated intra-abdominal infections, complicated urinary tract infections, nosocomial pneumonia and VAP.

\section{CONCLUSION}

In patients with nosocomial infections, appropriate initial antibiotic therapy is associated with higher survival rates, shorter hospital stays and lower healthcare costs. Broadspectrum antibiotics are an optimal initial choice for nosocomial pneumonia and severe sepsis. This represents an important paradigm shift from the traditional approach, which called for initial use of narrow-spectrum agents. The priority in treating severe infections is the patient. The best antibiotic should be prescribed first, based on the patient's risk factors, suspected pathogen and local resistance patterns. It is clearly very important to have open communication between the clinical laboratory and attending physicians about any changes in local pathogen epidemiology. In general, the maximal inflammatory response usually resolves over the first $48 \mathrm{~h}$. Accordingly, de-escalation [43] in antibiotic therapy, performed via simplification of the number of agents or reduction of the spectrum, should be undertaken based on both microbiological susceptibility data and clinical response.

\section{REFERENCES}

1 Kollef MH, Fraser VJ. Antibiotic resistance in the intensive care unit. Ann Intern Med 2001; 134: 298-314.

2 National Nosocomial Infections Surveillance System. National Nosocomial Infections Surveillance (NNIS) System Report, data summary from January 1992 through June 2004, issued October 2004. Am J Infect Control 2004; 32: 470-485.

3 Combes A, Luyt CE, Fagon JY, Wolff M, Trouillet JL, Chastre J. Impact of piperacillin resistance on the outcome of Pseudomonas ventilator-associated pneumonia. Intensive Care Med 2006; 32: 1970-1978.

4 Shorr AF, Combes A, Kollef MH, Chastre J. Methicillinresistant Staphylococcus aureus prolongs intensive care unit stay in ventilator-associated pneumonia, despite initially appropriate antibiotic therapy. Crit Care Med 2006; 34: 700-706.

5 Lautenbach E, Patel JB, Bilker WB, Edelstein PH, Fishman NO. Extended-spectrum $\beta$-lactamase-producing Escherichia coli and Klebsiella pneumoniae: risk factors for infection and impact of resistance on outcomes. Clin Infect Dis 2001; 32: 1162-1171.

6 Cosgrove SE, Kaye KS, Eliopoulous GM, Carmeli Y. Health and economic outcomes of the emergence of thirdgeneration cephalosporin resistance in Enterobacter species. Arch Intern Med 2002; 162: 185-190.

7 Tumbarello M, Spanu T, Sanguinetti M, et al. Bloodstream infections caused by extended-spectrum- $\beta$-lactamaseproducing Klebsiella pneumoniae: risk factors, molecular epidemiology, and clinical outcome. Antimicrob Agents Chemother 2006; 50: 498-504.
8 Kollef MH, Sherman G, Ward S, Fraser VJ. Inadequate antimicrobial treatment of infections: a risk factor for hospital mortality among critically ill patients. Chest 1999; 115: 462-474.

9 Alvarez-Lerma F. Modification of empiric antibiotic treatment in patients with pneumonia acquired in the intensive care unit. ICU-Acquired Pneumonia Study Group. Intensive Care Med 1996; 22: 387-394.

10 Rello J, Gallego M, Mariscal D, Soñora R, Valles J. The value of routine microbial investigation in ventilatorassociated pneumonia. Am J Respir Crit Care Med 1997; 156: 196-200.

11 Luna CM, Vujacich P, Niederman MS, et al. Impact of BAL data on the therapy and outcome of ventilator-associated pneumonia. Chest 1997; 111: 676-685.

12 Ibrahim EH, Sherman G, Ward S, Fraser VJ, Kollef MH. The influence of inadequate antimicrobial treatment of bloodstream infections on patient outcomes in the ICU setting. Chest 2000; 118: 146-155.

13 Vallés J, Rello J, Ochagavia A, Garnacho J, Alcalá MA, for the Spanish Collaborative Group for Infections in Intensive Care Units of Sociedad Española de Medicina Intensiva, Crítica y Unidades Coronarias. Community-acquired bloodstream infection in critically ill adult patients: impact of shock and inappropriate antibiotic therapy on survival. Chest 2003; 123: 1615-1624.

14 Garnacho-Montero J, Garcia-Garmendia JL, Barrero-Almodovar A, Jimenez-Jimenez FJ, Perez-Paredes C, OrtizLeyba C. Impact of adequate empirical antibiotic therapy on the outcome of patients admitted to the intensive care unit with sepsis. Crit Care Med 2003; 31: 2742-2751.

15 Pea F, Viale P. The antimicrobial therapy puzzle: could pharmacokinetic-pharmacodynamic relationships be helpful in addressing the issue of appropriate pneumonia treatment in critically ill patients? Clin Infect Dis 2006; 42: 1764-1771.

16 Rello J, Mallol J. Optimal therapy for methicillin-resistant Staphylococcus aureus pneumonia: what is the best dosing regimen? Chest 2006; 130: 938-940.

17 Chastre J, Trouillet JL. Problem pathogens (Pseudomonas aeruginosa and Acinetobacter). Semin Respir Infect 2000; 15: 287-298.

18 Baraibar J, Correa H, Mariscal D, Gallego M, Valles J, Rello J. Risk factors for infection by Acinetobacter baumannii in intubated patients with nosocomial pneumonia. Chest 1997; 112: 1050-1054.

19 Rello J, Torres A, Ricart M, et al. Ventilator-associated pneumonia by Staphylococcus aureus. Comparison of methicillin-resistant and methicillin-sensitive episodes. Am J Respir Crit Care Med 1994; 150: 1545-1549.

20 American Thoracic Society; Infectious Diseases Society of America. Guidelines for the management of adults with hospital-acquired, ventilator-associated, and healthcareassociated pneumonia. Am J Respir Crit Care Med 2005; 171: 388-416.

21 Wunderink RG, Rello J, Cammarata SK, Croos-Dabrera RV, Kollef MH. Linezolid versus vancomycin: analysis of two double-blind studies of patients with methicillin-resistant Staphylococcus aureus nosocomial pneumonia. Chest 2003; 124: 1789-1797. 
22 Richards MJ, Edwards JR, Culver DH, Gaynes RP. Nosocomial infections in medical intensive care units in the United States. National Nosocomial Infections Surveillance System. Crit Care Med 1999; 27: 887-892.

23 Rello J, Diaz E. Pneumonia in the intensive care unit. Crit Care Med 2003; 31: 2544-2551.

24 Colodner R. Extended-spectrum $\beta$-lactamases: a challenge for clinical microbiologists and infection control specialists. Am J Infect Control 2005; 33: 104-107.

25 Jones RN. Important and emerging $\beta$-lactamase-mediated resistances in hospital-based pathogens: the Amp C enzymes. Diagn Microbiol Infect Dis 1998; 31: 461-466.

26 Philippon A, Arlet G, Jacoby GA. Plasmid-determined AmpC-type $\beta$-lactamases. Antimicrob Agents Chemother 2002; 46: 1-11.

27 Paterson DL, Mulazimoglu L, Casellas JM, et al. Epidemiology of ciprofloxacin resistance and its relationship to extended-spectrum $\beta$-lactamase production in Klebsiella pneumoniae isolates causing bacteremia. Clin Infect Dis 2000; 30: $473-478$.

28 Babini GS, Livermore DM. Antimicrobial resistance amongst Klebsiella spp. collected from intensive care units in Southern and Western Europe in 1997-1998. J Antimicrob Chemother 2000; 45: 183-189.

29 Lautenbach E, Strom BL, Bilker WB, Patel JB, Edelstein PH, Fishman NO. Epidemiological investigation of fluoroquinolone resistance in infections due to extended-spectrum $\beta$-lactamase-producing Escherichia coli and Klebsiella pneumoniae. Clin Infect Dis 2001; 33: 1288-1294.

30 Trouillet JL, Vuagnat A, Combes A, Kassis N, Chastre J, Gibert C. Pseudomonas aeruginosa ventilator-associated pneumonia: comparison of episodes due to piperacillinresistant versus piperacillin-susceptible organisms. Clin Infect Dis 2002; 34: 1047-1054.

31 Colardyn F. Appropriate and timely empirical antimicrobial treatment of ICU infections-a role for carbapenems. Acta Clin Belg 2005; 60: 51-62.

32 Kollef MH. Appropriate empiric antimicrobial therapy of nosocomial pneumonia: the role of the carbapenems. Respir Care 2004; 49: 1530-1541.

33 Garcia-Rodriguez JA, Jones RN; MYSTIC Programme Study Group. Antimicrobial resistance in gram-negative isolates from European intensive care units: data from the Meropenem Yearly Susceptibility Test Information Collection (MYSTIC) programme. J Chemother 2002; 14: 25-32.

34 Rello J, Sa-Borges M, Correa H, Leal SR, Baraibar J. Variations in etiology of ventilator-associated pneumonia across four treatment sites: implications for antimicrobial prescribing practices. Am J Respir Crit Care Med 1999; 160: 608-613.

35 Vidaur L, Sirgo G, Rodriguez AH, Rello J. Clinical approach to the patient with suspected ventilator-associated pneumonia. Respir Care 2005; 50: 965-974.

36 Sandiumenge A, Diaz E, Bodi M, Rello J. Therapy of ventilator-associated pneumonia. A patient-based approach based on the ten rules of "The Tarragona Strategy". Intensive Care Med 2003; 29: 876-883.

37 Doan TL, Fung HB, Mehta D, Riska PF. Tigecycline: a glycylcycline antimicrobial agent. Clin Ther 2006; 28: 1079-1106.

38 Chambers HF. Ceftobiprole: in vivo profile of a bactericidal cephalosporin. Clin Microbiol Infect 2006; 12: Suppl. 2, S17-S22.

39 Jones R, Stilwell MG, Sader HS, Fritsche TR. Uniformly enhanced activity of doripenem compared to other carbapenems (imipenem, meropenem) when testing $P$. aeruginosa isolates: results from three continents. Int J Infect Dis 2006; 10: Suppl. 1, S127-S128.

40 Sakyo S, Tomita H, Tanimoto K, Fujimoto S, Ike Y. Potency of carbapenems for the prevention of carbapenem-resistant mutants of Pseudomonas aeruginosa: the high potency of a new carbapenem doripenem. J Antibiot (Tokyo) 2006; 59: 220-228.

41 Mushtaq S, Ge Y, Livermore DM. Doripenem versus Pseudomonas aeruginosa in vitro: activity against characterized isolates, mutants, and transconjugants and resistance selection potential. Antimicrob Agents Chemother 2004; 48: 3086-3092

42 Horiuchi M, Kimura M, Tokumura M, Hasebe N, Arai T, Abe K. Absence of convulsive liability of doripenem, a new carbapenem antibiotic, in comparison with $\beta$-lactam antibiotics. Toxicology 2006; 222: 114-124.

43 Lisboa T, Rello J. De-escalation in lower respiratory tract infections. Curr Opin Pulm Med 2006; 12: 364-368. 